\title{
The heat kernel coefficients for the dielectric cylinder
}

\author{
M. BORDAG* \\ University of Leipzig, Institute for Theoretical Physics \\ Augustusplatz 10/11, 04109 Leipzig, Germany \\ and I. G. Pirozhenko ${ }^{\dagger}$ \\ Bogolyubov Laboratory of Theoretical Physics, \\ Joint Institute for Nuclear Research, 141980 Dubna, Russia
}

June 24, 2021

\begin{abstract}
We calculate the heat kernel coefficients for the electromagnetic field in the background of a dielectric cylinder with non equal speeds of light inside and outside. The coefficient $a_{2}$ whose vanishing makes the vacuum energy of a massless field unique, turns out to be zero in dilute order, i.e., in order $(\varepsilon-1)^{2}$, and nonzero beyond. As a consequence, the vanishing of the vacuum energy in the presence of a dielectric cylinder found by Casimir-Polder summation must take place irrespectively of the methods by which it might be calculated.
\end{abstract}

\section{Introduction}

The vacuum energy of the electromagnetic field in the presence of a dielectric body is a topic of considerable interest since Schwingers attempt to explain sonoluminescence. Although this seems unlikely to be possible, mainly due

*e-mail: Michael.Bordag@itp.uni-leipzig.de

†e-mail: pirozhen@thsun1.jinr.ru 
to different time scales involved, open questions connected with the renormalization remain.

The divergent part of the vacuum energy can be expressed in terms of the corresponding heat kernel coefficients. By means of the renormalization procedure the divergent part must be subtracted from the ground state energy and added as counter term to the classical energy of the background. This general procedure does not work straightforwardly for a dielectric body. The point is that there is no classical energy which may be associated with it. Note that there are no electric or magnetic fields present, instead there is merely the ability of the body to get polarized upon application of an electromagnetic field. One may overcome this problem by introducing formally the necessary terms for a classical energy and put zero the coefficients in front of them. This is similar to semiclassical gravity where one assumes the coefficients in front of the terms quadratic in curvature to be sufficiently small. But there is one more problem associated with the vacuum energy of a dielectric body. As the field is massless, there is no normalization condition to get a unique ground state energy as long as the heat kernel coefficient $a_{2}$ is nonzero because it comes with the logarithmic divergence, see [1] for a detailed explanation.

In [1] the heat kernel coefficients for a generic smooth dielectric background and for a dielectric ball have been calculated. The coefficient $a_{2}$ turned out to be nonzero in general. For small deviation of the dielectricity $\varepsilon$ from unity (resp. from its value at spatial infinity) $a_{2}$ is zero to first order in $(\varepsilon-1)$ and nonzero starting from second order, $(\varepsilon-1)^{2}$, which is called dilute approximation. For the dielectric ball it is zero in second order in addition. In this way, for a more singular background configuration $(\varepsilon(r)=\varepsilon \Theta(R-r)$ has a jump) the singularity is slightly weaker. It is this zero of $a_{2}$ to order $(\varepsilon-1)^{2}$ which allowed to get a unique result for the vacuum energy of the dielectric ball in dilute approximation,

$$
\mathcal{E}_{0}^{\text {ball }}=\frac{23}{1536 \pi} \frac{(\varepsilon-1)^{2}}{R}+O\left((\varepsilon-1)^{3}\right),
$$

which was obtained in by Casimir-Polder summation in [2], by a perturbative setup in [3] and by mode summation in [4].

In the present paper we consider a dielectric cylinder and calculate the heat kernel coefficients $a_{n}$ for $n \leq 2$. This problem is technically more involved than the corresponding calculation for the ball. Although the Jost function of the corresponding scattering problem which we use is expressed 
in terms of Bessel functions the photon polarizations do not separate and there is an additional dependence on the axial momentum.

The vacuum energy of a dielectric cylinder had been calculated in [5] in dilute approximation by summing the Casimir-Polder potentials and it turned out to be zero,

$$
\mathcal{E}_{0}^{\mathrm{cyl}}=O\left((\varepsilon-1)^{3}\right) .
$$

In the present paper we show that the corresponding $a_{2}$ is zero in this (dilute) approximation and conclude that the vacuum energy in dilute order is zero irrespectively of the method by which it is calculated.

In this paper we use units with $\hbar=c=1$.

\section{Basic formulas}

In order to regularize the vacuum energy of the electromagnetic field we use the zeta functional regularization and express the energy in terms of the corresponding zeta function,

$$
\mathcal{E}_{0}(s)=\frac{\mu^{2}}{2} \zeta\left(s-\frac{1}{2}\right) .
$$

In the presence of the dielectric cylinder the spectrum of the electromagnetic field is completely continuous and we use the representation of the regularized ground state energy derived in [6] for a spherically symmetric background which was in [7] rewritten for a cylindrically symmetric background. After dropping the Minkowski space contribution we obtain

$$
\zeta(s)=\frac{\sin (\pi s)}{\pi} \sum_{l=-\infty}^{\infty} \int_{0}^{\infty} \frac{d k_{z}}{\pi} \int_{k_{z}}^{\infty} d k\left(k^{2}-k_{z}^{2}\right)^{-s} \frac{\partial}{\partial k} \ln \Delta_{l}\left(k, k_{z}\right) .
$$

Here, $\Delta_{l}\left(k, k_{z}\right)$ is the Jost function for the scattering of electromagnetic waves off the cylinder with radius $R$ on the imaginary axis. It is given by

$$
\begin{aligned}
\Delta_{l}\left(k, k_{z}\right)= & \frac{1}{\Delta_{l}^{\infty}}\left\{\Delta_{l}^{T M}\left(k, k_{z}\right) \Delta_{l}^{T E}\left(k, k_{z}\right)\right. \\
& \left.+l^{2}\left(k^{2}-k_{z}^{2}\right) k_{z}^{2}\left(1-\alpha^{2}\right)^{2} c_{2}^{-2}\left[I_{l}(q R) K_{l}(k R)\right]^{2}\right\}
\end{aligned}
$$

with

$$
\Delta_{l}^{T M}\left(k, k_{z}\right)=\mu_{1} k I_{l}^{\prime}(q R) K_{l}(k R)-\mu_{2} q I_{l}(q R) K_{l}^{\prime}(k R),
$$




$$
\begin{aligned}
\Delta_{l}^{T E}\left(k, k_{z}\right) & =\varepsilon_{1} k I_{l}^{\prime}(q R) K_{l}(k R)-\varepsilon_{2} q I_{l}(q R) K_{l}^{\prime}(k R) \\
\Delta_{l}^{\infty}\left(k, k_{z}\right) & =\frac{1}{4} e^{2(q-k)} q k\left(\varepsilon_{1} k+\varepsilon_{2} q\right)\left(\mu_{1} k+\mu_{2} q\right)
\end{aligned}
$$

and the notations $q=\sqrt{\alpha^{2} k^{2}+k_{z}^{2}\left(1-\alpha^{2}\right)}, \alpha \equiv c_{2} / c_{1}$; and the speeds of light inside and outside are $c_{i}=1 / \sqrt{\varepsilon_{i} \mu_{i}}(i=1,2)$. This formula can be found in textbooks on classical electrodynamics, see also [8].

The heat kernel coefficients are known to be defined by the residues of the zeta function at the corresponding points $s$,

$$
a_{n}=(4 \pi)^{3 / 2} \operatorname{Res}_{s=\frac{3}{2}-n} \Gamma(s) \zeta(s) .
$$

These residua result from large all, the momentum $k$, the axial momentum $k_{z}$ and the orbital momentum $l$. To factorize the orbital momentum dependence for the terms with $l \neq 0$ we use the uniform asymptotic expansion of the Bessel functions [9]. Being inserted into Eq. (5) it gives raise to the expansion

$$
\ln \Delta_{l}\left(l k, l k_{z}\right) \sim \sum_{i=-1,0,1, \ldots} \frac{D_{i}\left(k, k_{z}\right)}{l^{i}}
$$

for $k, l \rightarrow \infty$. Substituting the latter into (4) previously changing the variables $k \rightarrow l k$ and $k_{z} \rightarrow l k_{z}$ so that $k$ and $k_{z}$ were fixed, we split the zeta function into parts according to

$$
\Gamma(s) \zeta_{c y l}(s)=\tilde{A}(s)+2\left\{A_{-1}(s)+A_{0}(s)+A_{1}(s)+A_{2}(s)+A_{3}(s)+\ldots\right\} .
$$

Here $\tilde{A}(s)$ results from $l=0$

$$
\tilde{A}(s)=\frac{R^{2 s-1}}{c_{2}^{2 s} \Gamma(1-s)} \int_{0}^{\infty} \frac{d k_{z}}{\pi} \int_{k_{z}}^{\infty} d k\left(k^{2}-k_{z}^{2}\right)^{-s} \frac{\partial}{\partial k} \ln \Delta_{0}\left(k, k_{z}\right),
$$

and below we will use the asymptotic expansion of the Bessel functions for large argument in it. The $A_{i}(s)$ 's are generated by the expansion (8)

$$
A_{i}(s)=\frac{R^{2 s-1} \zeta_{R}(2 s-1+i)}{c_{2}^{2 s} \Gamma(1-s)} \int_{0}^{\infty} \frac{d k_{z}}{\pi} \int_{k_{z}}^{\infty} d k\left(k^{2}-k_{z}^{2}\right)^{-s} \frac{\partial}{\partial k} \ln D_{i}\left(k, k_{z}\right) .
$$

For the heat kernel coefficients up to $a_{2}$ it is sufficient to include $i$ up to 3 . The summation over $l$ (from 1 to $\infty$ ) was carried out in $A_{i}(s)$ and resulted in the Riemann zeta function. 
The expressions for the $D_{i}$ are simple for $i=-1$ and $i=0$, but sufficiently involved for $i=1,2,3$ to be banned into the appendix. Here we note

$$
\begin{aligned}
D_{-1}= & 2\left(q-k+\sqrt{1+q^{2}}-\sqrt{1+k^{2}}+\ln \frac{q}{k} \frac{1+\sqrt{1+q^{2}}}{1+\sqrt{1+k^{2}}}\right), \\
D_{0}= & \ln \left\{\left[\mu_{1} \frac{k}{q}\left(\frac{1+q^{2}}{1+k^{2}}\right)^{1 / 4}+\mu_{2} \frac{q}{k}\left(\frac{1+k^{2}}{1+q^{2}}\right)^{1 / 4}\right] \times\left[\mu_{i} \leftrightarrow \varepsilon_{i}\right]\right. \\
& \left.+\frac{\left(k^{2}-k_{z}^{2}\right)\left(1-\alpha^{2}\right)^{2} k_{z}^{2}}{c_{2}^{2} k^{2} q^{2} \sqrt{1+q^{2}} \sqrt{1+k^{2}}}\right\}+\ln \left\{\frac{q k}{\left(\varepsilon_{1} k+\varepsilon_{2} q\right)\left(\mu k+\mu_{2} q\right)}\right\} .
\end{aligned}
$$

In the next section the functions $A_{i}(i=-1, . .3)$ and $\tilde{A}(s)$ are considered one after another in order to find their residues contributing to the heatkernel coefficients.

\section{Calculation of the heat-kernel coefficients}

In this section we give a detailed calculation of the heat-kernel coefficient $a_{2}$. The results of the analogous calculations for the junior coefficients $a_{n}$ with $n<2$ are listed in the end of the section.

We start from $A_{-1}(s)$, where it is easy to carry out the integration explicitly

$$
A_{-1}(s)=-\frac{R^{2 s-1} \zeta(2 s-2)}{c_{2}^{2 s} \pi}\left(\alpha^{2 s}-1\right) \frac{\Gamma(s-1)}{1-2 s} .
$$

We are interested in the residue of $A_{-1}(s)$ at the point $s=-1 / 2$. As $A_{-1}$ has no pole at this point, the residue equals zero. So, this term of the expansion (9) doesn't contribute to $a_{2}$.

The term $A_{0}(s)$ is more complicated. To simplify the integration we make the change of variables $k \rightarrow k, k_{z} \rightarrow k \eta$. It gives

$$
\begin{aligned}
A_{0}(s)= & \frac{\zeta(2 s-1)}{\pi} \frac{R^{2 s-1}}{c_{2}^{2 s} \Gamma(1-s)} \\
& \int_{0}^{1} d \eta\left(1-\eta^{2}\right)^{-s} \int_{0}^{\infty} d k k^{-2 s+1}\left[\frac{\partial}{\partial k}-\frac{\eta}{k} \frac{\partial}{\partial \eta}\right] D_{0}(k, \eta) .
\end{aligned}
$$

The function

$$
D_{0}(k, \eta)=\ln \left\{\left[\mu_{1}\left(\frac{1+\gamma^{2} k^{2}}{1+k^{2}}\right)^{1 / 4}+\mu_{2} \gamma^{2}\left(\frac{1+k^{2}}{1+\gamma^{2} k^{2}}\right)^{1 / 4}\right] \times\left[\mu_{i} \leftrightarrow \varepsilon_{i}\right]\right.
$$




$$
\left.+\frac{\left(1-\eta^{2}\right)\left(1-\alpha^{2}\right)^{2} \eta^{2}}{c_{2}^{2} \sqrt{1+\gamma^{2} k^{2}} \sqrt{1+k^{2}}}\right\}+\ln \frac{1}{\gamma\left(\varepsilon_{1}+\varepsilon_{2} \gamma\right)\left(\mu_{1}+\mu_{2} \gamma\right)},
$$

with $\gamma=\sqrt{\alpha^{2}+\left(1-\alpha^{2}\right) \eta^{2}}$, has the following asymptotics at zero and infinity,

$$
\begin{aligned}
\left.D_{0}(k, \eta)\right|_{k \rightarrow 0}= & \ln \frac{\left(\mu_{1}+\gamma^{2} \mu_{2}\right)\left(\varepsilon_{1}+\gamma^{2} \varepsilon_{2}\right)+\left(1-\eta^{2}\right) \eta^{2}\left(1-\alpha^{2}\right)^{2} c_{2}^{-2}}{\gamma\left(\varepsilon_{1}+\varepsilon_{2} \gamma\right)\left(\mu_{1}+\mu_{2} \gamma\right)} \\
& +\mathcal{O}\left(k^{2}\right), \\
\left.D_{0}(k, \eta)\right|_{k \rightarrow \infty}= & \frac{\left(1-\alpha^{2}\right)^{2} \eta^{2}\left(1-\eta^{2}\right)}{2 c_{2}^{2} \gamma^{2}\left(\mu_{1}+\mu_{2} \gamma\right)\left(\varepsilon_{1}+\varepsilon_{2} \gamma\right)} \frac{1}{k^{2}}+\mathcal{O}\left(k^{-3}\right) .
\end{aligned}
$$

Therefore the integral over $k$ in $A_{0}$ converges in the range $-1 / 2<s<1 / 2$. Adding and subtracting from the integrand its asymptotics at $k \rightarrow \infty$ we obtain the analytic continuation into the vicinity of the point $s=-1 / 2$

$$
\begin{gathered}
A_{0}(s)=\frac{\zeta(2 s-1)}{\pi} \frac{R^{2 s-1}}{c_{2}^{2 s} \Gamma(1-s)} \int_{0}^{1} d \eta\left(1-\eta^{2}\right)^{-s} \\
\quad \times\left\{\int_{0}^{\infty} \frac{d k}{k^{2 s-1}}\left[\frac{\partial}{\partial k}-\frac{\eta}{k} \frac{\partial}{\partial \eta}\right]\left[D_{0}(k, \eta)-\frac{\eta^{2}\left(1-\eta^{2}\right)\left(1-\alpha^{2}\right)^{2}}{2 c_{2}^{2} \gamma^{2}\left(\mu_{1}+\mu_{2} \gamma\right)\left(\varepsilon_{1}+\varepsilon_{2} \gamma\right)} \frac{1}{(k+1)^{2}}\right]\right. \\
\left.\quad-\frac{1}{2}\left[(-2 s+1)+\eta \frac{\partial}{\partial \eta}\right] \Gamma(-2 s+1) \Gamma(2 s+1) \frac{\eta^{2}\left(1-\eta^{2}\right)\left(1-\alpha^{2}\right)^{2}}{c_{2}^{2} \gamma^{2}\left(\mu_{1}+\mu_{2} \gamma\right)\left(\varepsilon_{1}+\varepsilon_{2} \gamma\right)}\right\} .
\end{gathered}
$$

The first integral is now analytical around $s=-1 / 2$, the second one contains the pole. But this pole is canceled by the Riemann zeta function $\zeta(2 s-1)$ which equals zero at $s=-1 / 2$. As a result we have

$$
\underset{s \rightarrow-1 / 2}{\operatorname{res}} A_{0}^{\text {sing }}(s)=0 .
$$

Analyzing now the asymptotics of $D_{1}(s)$ at zero and at infinity,

$$
\left.D_{1}\right|_{k \rightarrow 0} \rightarrow \frac{1}{2}\left(\gamma^{2}-1\right) k^{2}+\mathcal{O}\left(k^{4}\right),\left.\quad D_{1}\right|_{k \rightarrow \infty} \rightarrow \frac{\Theta_{1}}{k}+\mathcal{O}\left(k^{-3}\right),
$$

where

$$
\Theta_{1}=\frac{1}{4}\left\{3-\frac{3}{\gamma}+2 \frac{\varepsilon_{2}-\varepsilon_{1}}{\varepsilon_{1}+\varepsilon_{2} \gamma}+\frac{\mu_{2}-\mu_{1}}{\mu_{1}+\mu_{2} \gamma}\right\}
$$


one comes to the conclusion that the integral over $k$ in

$$
A_{1}(s)=\frac{\zeta(2 s)}{\pi} \frac{R^{2 s-1}}{c_{2}^{2 s} \Gamma(1-s)} \int_{0}^{1} d \eta\left(1-\eta^{2}\right)^{-s} \int_{0}^{\infty} d k k^{-2 s+1}\left[\frac{\partial}{\partial k}-\frac{\eta}{k} \frac{\partial}{\partial \eta}\right] D_{1}(k, \eta),
$$

converges in the strip $0<s<3 / 2$. The analytic continuation to the point $s=-1 / 2$ is the following

$$
\begin{aligned}
A_{1}(s)= & \zeta(2 s) \frac{c_{2}^{-2 s} R^{2 s-1}}{\pi \Gamma(1-s)} \\
& \times\left\{\int_{0}^{1} \frac{d \eta}{\left(1-\eta^{2}\right)^{s}} \int_{0}^{\infty} d k k^{-2 s+1}\left\{\frac{\partial}{\partial k}-\frac{\eta}{k} \frac{\partial}{\partial \eta}\right\}\left[D_{1}(k, \eta)-\frac{\Theta_{1}(\eta)}{\sqrt{k^{2}+1}}\right]\right. \\
& \left.-\Gamma(s) \int_{0}^{1} \frac{d \eta}{\left(1-\eta^{2}\right)^{s}}\left[\Gamma(-s+3 / 2)+\frac{\Gamma(-s+1 / 2)}{2} \eta \frac{\partial}{\partial \eta}\right] \Theta_{1}(\eta)\right\} .
\end{aligned}
$$

Here the asymptotics of $D_{1}(k, \eta)$ for $k \rightarrow \infty$ was added and subtracted. Both terms in (17) are analytic around $s=-1 / 2$. Therefore $\underset{s \rightarrow-1 / 2}{\operatorname{res}} A_{1}^{\text {sing }}(s)=0$.

Similar arguments for $A_{2}(s)$, where the integral with respect to $k$ exists at $-1 / 2<s<3 / 2$, give

$$
\begin{aligned}
\underset{s \rightarrow-1 / 2}{\operatorname{res}} A_{2}(s)= & -\frac{R^{-2}}{32} \frac{c_{2}}{\pi^{3 / 2}} \int_{0}^{1} \frac{d \eta}{\sqrt{1-\eta^{2}}} \\
& \times\left[\frac{3 \mu_{2}^{2} \gamma^{4}-\mu_{2}^{2} \gamma^{2}-4 \gamma^{2} \mu_{1} \mu_{2}+3 \mu_{1}^{2}-\gamma^{2} \mu_{1}^{2}}{\gamma^{2}\left(\mu_{1}+\mu_{2}\right)^{2}}+(\mu \leftrightarrow \varepsilon)\right] .
\end{aligned}
$$

The next term in (9) we have to consider is $A_{3}(s)$. The integrals in $A_{3}(s)$ converge at $s=-1 / 2$, while the Riemann zeta function has a pole at this point. Thus

$$
\underset{s \rightarrow-1 / 2}{\operatorname{res}} A_{3}(s)=\frac{R^{-2} c_{2}}{\pi^{3 / 2}} \int_{0}^{1} d \eta \sqrt{1-\eta^{2}} \int_{0}^{\infty} d k k^{2}\left[\frac{\partial}{\partial k}-\frac{\eta}{k} \frac{\partial}{\partial \eta}\right] D_{3} .
$$

Now we are left with the term $\tilde{A}(s)$ in Eq. (10).

$$
\tilde{A}(s)=\frac{R^{2 s-1}}{c_{2}^{2 s} \pi \Gamma(1-s)} \int_{0}^{1} d \eta\left(1-\eta^{2}\right)^{-s} \int_{0}^{\infty} d k k^{-2 s+1}\left[\frac{\partial}{\partial k}-\frac{\eta}{k} \frac{\partial}{\partial \eta}\right] \ln \Delta_{0}(k, \eta) .
$$


The asymptotic behavior of $\ln \Delta_{0}(k, \eta)$ at $k=0$ and $k=\infty$ is the following

$$
\begin{aligned}
\left.\ln \tilde{\Delta}(k, \eta)\right|_{k=0}= & \ln \frac{4 \varepsilon_{2} \mu_{2} \gamma^{3}}{\left(\varepsilon_{1}+\varepsilon_{2} \gamma\right)\left(\mu_{1}+\mu_{2} \gamma\right)}+2(1-\gamma) k+\mathcal{O}\left(k^{2}\right), \\
\left.\ln \tilde{\Delta}(k, \eta)\right|_{k=\infty}= & \frac{1}{8} \frac{-\varepsilon_{1} \gamma-3 \varepsilon_{1}+3 \varepsilon_{2} \gamma^{2}+\varepsilon_{2} \gamma}{\left(\varepsilon_{1}+\varepsilon_{2} \gamma\right) \gamma} \frac{1}{k} \\
& +\frac{-3 \varepsilon_{2}^{2} \gamma^{4}+4 \varepsilon_{1} \varepsilon_{2} \gamma^{2}+\varepsilon_{2}^{2} \gamma^{2}+\varepsilon_{1}^{2} \gamma^{2}-3 \varepsilon_{1}^{2}}{16 \gamma^{2}\left(\varepsilon_{1}+\varepsilon_{2} \gamma\right)^{2}} \frac{1}{k^{2}} \\
& +\left\{\varepsilon_{i} \leftrightarrow \mu_{i}\right\}+\mathcal{O}\left(k^{-3}\right) .
\end{aligned}
$$

The integrals over $k$ for the first an the second terms in the square brackets of (20) exist correspondingly in the ranges $0<s<1$ and $0<s<1 / 2$. We divide the integration with respect to $k$ in two parts $\int_{0}^{\infty}=\int_{0}^{1}+\int_{1}^{\infty}$, where the first one is regular at $s=-1 / 2$, and the second one diverges in the upper bound. Keeping in mind that only the singular part gives contribution to the residue we omit the term convergent at $s=-1 / 2$. To obtain the analytic continuation to the point $s=-1 / 2$ one should add and subtract from $\ln \tilde{\Delta}(k, \eta)$ two terms of its expansion in infinity. After that it is easy to find the residue

$$
\begin{aligned}
\underset{s \rightarrow-1 / 2}{\operatorname{ses}} \tilde{A}(s)= & \frac{c_{2} R^{-2}}{16 \pi^{3 / 2}} \int_{0}^{1} \frac{d \eta}{\sqrt{1-\eta^{2}}} \\
& \times\left[\frac{3 \mu_{2}^{2} \gamma^{4}-\mu_{2}^{2} \gamma^{2}-4 \gamma^{2} \mu_{1} \mu_{2}+3 \mu_{1}^{2}-\gamma^{2} \mu_{1}^{2}}{\gamma^{2}\left(\mu_{1}+\mu_{2} \gamma\right)^{2}}+(\mu \leftrightarrow \varepsilon)\right],
\end{aligned}
$$

which up to the multiplier $-1 / 2$ coincides with (18)

Summing up we see that only the functions $A_{2}, A_{3}$ and $\tilde{A}$ have nonzero residues in $s=-\frac{1}{2}$. But as the residues of $A_{2}$ and $\tilde{A}$ are mutually canceled in (9), the heat-kernel coefficient $a_{2}$ is totally defined by the contribution from $A_{3}$. The integration over $\eta$ and $k$ in terms of elementary functions seems impossible in (19). For $\mu_{i}=1$ simplifications occur, but still we are able to analyze the behavior of the coefficient $a_{2}$ as a function of the velocities if light only numerically (see Fig.1).

In the limit of small differences of the velocities of light (dilute dielectric cylinder) one might expand $\underset{s \rightarrow-1 / 2}{r e s} A_{3}(s)$ in powers of $c_{1}-c_{2}$. The expansion starts from the third order

$$
\underset{s \rightarrow-1 / 2}{\operatorname{res}} \zeta_{c y l}(s)=-\frac{9}{1408} \frac{R^{-2}}{\sqrt{\pi} c_{2}^{2}}\left(c_{1}-c_{2}\right)^{3}+\ldots
$$




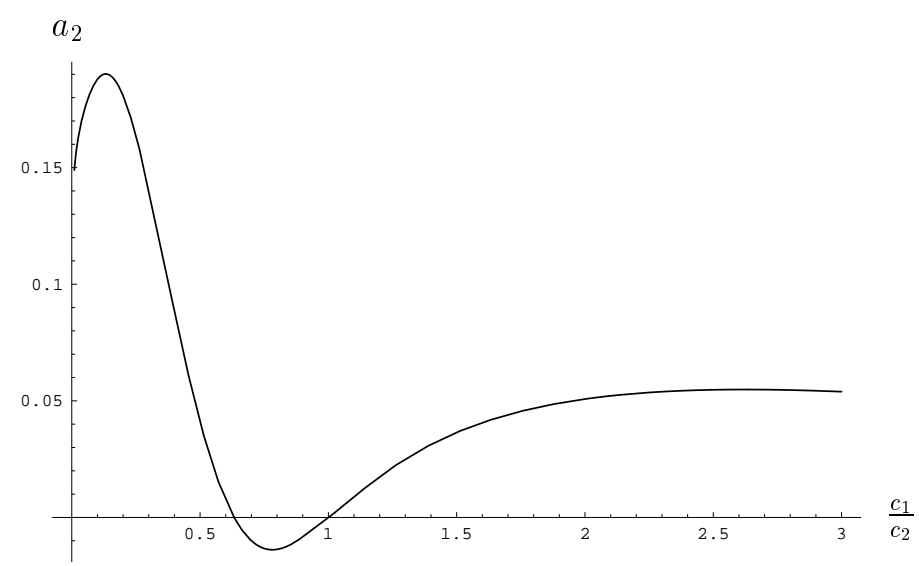

Figure 1: The heat kernel coefficient $a_{2}$ for the dielectric cylinder as function of the ration $c_{1} / c_{2}$ of the speeds of light inside vs. outside the cylinder

The heat kernel coefficient $a_{2}$ is

$$
a_{2}=-\frac{\pi}{R^{2} c_{2}^{2}} \frac{9}{88}\left(c_{1}-c_{2}\right)^{3}+\ldots
$$

The heat-kernel coefficients with $n<2$ are obtained in the same way. For arbitrary velocities of light inside and outside the cylinder one gets

$$
\begin{aligned}
& a_{0}=-\frac{4 \pi R^{2}}{c_{2}^{3}}\left(1-\alpha^{3}\right) \\
& a_{1 / 2}=-\frac{2 \pi^{3 / 2} R}{c_{2}^{4}} \frac{\left(1-\alpha^{2}\right)^{2}}{\left(\mu_{1}+\mu_{2}\right)\left(\varepsilon_{1}+\varepsilon_{2}\right)}, \\
& a_{1}=-\frac{2 \pi}{3 c_{2}}(1-\alpha)-\frac{8}{c_{2}} \int_{0}^{\infty} \frac{d k}{k} \int_{0}^{1} d \eta \frac{\eta}{\sqrt{1-\eta^{2}}} \frac{\partial}{\partial \eta} D_{1}(k, \eta), \\
&+\frac{4}{c_{2}} \int_{0}^{1} \frac{d \eta \eta}{\sqrt{1-\eta^{2}}} \\
& \times \frac{\partial}{\partial \eta} \ln \left[\frac{4 \gamma^{4} \varepsilon_{2} \mu_{2}}{\left(\mu_{1}+\mu_{2} \gamma^{2}\right)\left(\varepsilon_{1}+\varepsilon_{2} \gamma^{2}\right)+\left(1-\eta^{2}\right) \eta^{2}\left(1-\alpha^{2}\right)^{2} c_{2}^{-2}}\right], \\
& 3 \pi^{3 / 2} \frac{\varepsilon_{1}^{2} \mu_{1}^{2}-4 \varepsilon_{1} \mu_{1} \varepsilon_{2} \mu_{2}+\varepsilon_{2}^{2} \mu_{2}^{2}+\varepsilon_{1}^{2} \mu_{2}^{2}+\varepsilon_{2}^{2} \mu_{1}^{2}}{\left(\varepsilon_{1}+\varepsilon_{2}\right)^{2}\left(\mu_{1}+\mu_{2}\right)^{2}} . \\
& a_{3 / 2}=
\end{aligned}
$$




\section{Conclusions}

In the preceding sections we calculated the heat kernel coefficients $a_{n}$ with $n \leq 2$ of the electromagnetic field in the background of a dielectric cylinder for different speeds of light inside and outside. The coefficient $a_{2}$ vanishes in the dilute order.

The result of a non vanishing $a_{2}$ beyond the dilute order, obtained in [1] for a dielectric ball and here by Eq. (24) for a cylinder implies an yet unsolved problem. On the one hand side the vacuum energy of the electromagnetic field in the background of a dielectric body is a physical quantity and at least in principle measurable. On the other hand side even after removing the ultraviolet divergences by hand, it is not uniquely defined due to the arbitrariness resulting from the logarithmic divergence. The only explanation which seems meaningful states that the problem itself is ill defined. The setup of a material body characterized by a position dependent $\varepsilon(\vec{x})$, a step-like $\varepsilon(r)=\varepsilon \Theta(R-r)$ for instance, is clearly an idealization. Another example for an idealization are conductor boundary conditions (plane mirrors or a infinitely thin spherical shell for example) delivering a uniquely defined vacuum energy.

So this idealization does not work for a dielectric body. This is surprising in view of the whole area of electromagnetic phenomena like scattering of waves not connected with vacuum energy where it works well. In order to attack this problem one has probably to take some microscopic model for the dielectric body, a lattice of harmonic oscillators for instance and to calculate the vacuum energy in that background beyond the dilute approximation. Then one had to investigate a limiting procedure to turn to the continuous dielectric body. One should expect that a dependence of the vacuum energy on some parameter like lattice spacing or plasma frequency remains resulting in the corresponding logarithmic term connected with $a_{2}$. There are recent attempts to do so, see [10, 11, 12].

\section{Acknowledgments}

The work was supported by the Heisenberg-Landau Program and Russian Foundation for Basic Research (Grant No. 00-01-00300) 


\section{Appendix A}

In this appendix we give a list of functions entering the expansion (8). They are written in terms of the variables $k$ and $\eta$ with making use of the notations $t_{1}=1 / \sqrt{1+\gamma^{2} k^{2}}, \quad t_{2}=1 / \sqrt{1+k^{2}}, \quad \gamma=\sqrt{\alpha^{2}+\left(1-\alpha^{2}\right) \eta^{2}}$ and

$$
\begin{aligned}
& D_{1}(k, \eta)=\mathcal{F}_{1}, \\
& D_{2}(k, \eta)=-\frac{1}{2} \mathcal{F}_{1}^{2}+\mathcal{F}_{2}, \\
& D_{3}(k, \eta)=\mathcal{F}_{3}-\mathcal{F}_{1} \times \mathcal{F}_{2},
\end{aligned}
$$

where

$$
\begin{aligned}
\mathcal{F}_{1}= & \frac{1}{\mathcal{F}}\left\{\frac { ( 1 - t _ { 1 } ^ { 2 } ) ( 1 - t _ { 2 } ^ { 2 } ) } { t _ { 1 } ^ { 2 } t _ { 2 } ^ { 2 } } \left[\varepsilon_{1} \mu_{1} \frac{t_{1}\left(1-t_{2}^{2}\right)}{t_{2}\left(1-t_{1}^{2}\right)}\left(\frac{5}{12} t_{2}^{3}-\frac{1}{4} t_{2}-\frac{3}{4} t_{1}+\frac{7}{12} t_{1}^{3}\right)\right.\right. \\
& +\left(\varepsilon_{1} \mu_{2}+\varepsilon_{2} \mu_{1}\right)\left(-\frac{t_{2}^{3}}{12}+\frac{t_{2}}{4}-\frac{t_{1}}{4}+\frac{t_{1}^{3}}{12}\right) \\
& \left.+\varepsilon_{2} \mu_{2} \frac{t_{2}\left(1-t_{1}^{2}\right)}{t_{1}\left(1-t_{2}^{2}\right)}\left(\frac{3}{4} t_{2}-\frac{7}{12} t_{2}^{3}+\frac{t_{1}}{4} \frac{5}{12} t_{1}^{3}\right)\right] \\
& \left.+\varepsilon_{2} \mu_{2}\left(1-\eta^{2}\right) \eta^{2}\left(1-\alpha^{2}\right)^{2} t_{1} t_{2}\left(\frac{5}{12} t_{2}^{3}-\frac{t_{2}}{4}+\frac{t_{1}}{4}-\frac{5}{12} t_{1}^{3}\right)\right\}, \\
\mathcal{F}_{2}= & \frac{1}{\mathcal{F}}\left\{\frac { ( 1 - t _ { 1 } ^ { 2 } ) ( 1 - t _ { 2 } ^ { 2 } ) } { t _ { 1 } ^ { 2 } t _ { 2 } ^ { 2 } } \left[\varepsilon _ { 1 } \mu _ { 1 } \frac { t _ { 1 } ( 1 - t _ { 2 } ^ { 2 } ) } { t _ { 2 } ( 1 - t _ { 1 } ^ { 2 } ) } \left(\frac{3}{16} t_{1} t_{2}-\frac{5}{16} t_{1} t_{2}^{3}-\frac{41}{48} t_{2}^{4}\right.\right.\right. \\
& \left.-\frac{7}{48} t_{1}^{3} t_{2}+\frac{35}{144} t_{1}^{3} t_{2}^{3}+\frac{5}{32} t_{2}^{2}+\frac{205}{288} t_{2}^{6}+\frac{13}{16} t_{1}^{4}-\frac{203}{288} t_{1}^{6}-\frac{3}{32} t_{1}^{2}\right) \\
& +\left(\varepsilon_{1} \mu_{2}+\varepsilon_{2} \mu_{1}\right)\left(-\frac{1}{16} t_{1} t_{2}+\frac{1}{48} t_{1} t_{2}^{3}+\frac{11}{48} t_{2}^{4}+\frac{1}{48} t_{1}^{3} t_{2}-\frac{1}{144} t_{1}^{3} t_{2}^{3}\right. \\
& \left.-\frac{3}{32} t_{2}^{2}-\frac{35}{288} t_{2}^{6}+\frac{11}{48} t_{1}^{4}-\frac{35}{288} t_{1}^{6}-\frac{3}{32} t_{1}^{2}\right) \\
& +\varepsilon_{2} \mu_{2} \frac{t_{2}\left(1-t_{1}^{2}\right)}{t_{1}\left(1-t_{2}^{2}\right)}\left(\frac{3}{16} t_{1} t_{2}-\frac{7}{48} t 1 t_{2}^{3}+\frac{13}{16} t_{2}^{4}-\frac{5}{16} t_{1}^{3} t_{2}+\frac{35}{144} t_{1}^{3} t_{2}^{3}\right. \\
& \left.\left.-\frac{3}{32} t_{2}^{2}-\frac{203}{288} t_{2}^{6}-\frac{41}{48} t_{1}^{4}+\frac{205}{288} t_{1}^{6}+\frac{5}{32} t_{1}^{2}\right)\right] \\
& +\varepsilon_{2} \mu_{2}\left(1-\eta^{2}\right) \eta^{2}\left(1-\alpha^{2}\right)^{2} t_{1} t_{2}\left(-\frac{1}{16} t_{1} t_{2}+\frac{5}{48} t_{1} t_{2}^{2}-\frac{41}{48} t_{2}^{4}\right. \\
& \\
&
\end{aligned}
$$




$$
\begin{aligned}
& +\frac{5}{48} t_{1}^{3} t_{2}-\frac{25}{144} t_{1}^{3} t_{2}^{3}+\frac{5}{32} t_{2}^{2}+\frac{205}{288} t_{2}^{6}-\frac{41}{48} t_{1}^{4}+\frac{205}{288} t_{1}^{6} \\
& \left.\left.+\frac{5}{32} t_{1}^{2}\right)\right\} \\
& \mathcal{F}_{3}=\frac{1}{\mathcal{F}}\left\{\frac { ( 1 - t _ { 1 } ^ { 2 } ) ( 1 - t _ { 2 } ^ { 2 } ) } { t _ { 1 } ^ { 2 } t _ { 2 } ^ { 2 } } \left[\varepsilon _ { 1 } \mu _ { 1 } \frac { t _ { 1 } ( 1 - t _ { 2 } ^ { 2 } ) } { t _ { 2 } ( 1 - t _ { 1 } ^ { 2 } ) } \left(-\frac{5}{128} t_{1} t_{2}^{2}+\frac{41}{64} t_{1} t_{2}^{4}\right.\right.\right. \\
& -\frac{205}{384} t_{1} t_{2}^{6}+\frac{203}{1152} t_{1}^{6} t_{2}-\frac{1015}{3456} t_{1}^{6} t_{2}^{3}-\frac{13}{64} t_{1}^{4} t_{2}+\frac{65}{192} t_{1}^{4} t_{2}^{3} \\
& +\frac{1435}{3456} t_{1}^{3} t_{2}^{6}+\frac{3}{128} t_{1}^{2} t_{2}-\frac{5}{128} t_{1}^{2} t_{2}^{3}-\frac{21}{128} t_{2}^{3}+\frac{3671}{1920} t_{2}^{5} \\
& -\frac{4543}{1152} t_{2}^{7}+\frac{22715}{10368} t_{2}^{9}+\frac{35}{384} t_{1}^{3} t_{2}^{2}-\frac{287}{576} t_{1}^{3} t_{2}^{4}+\frac{21385}{10368} t_{1}^{9} \\
& \left.-\frac{15}{128} t_{1}^{3}-\frac{469}{128} t_{1}^{7}+\frac{1103}{640} t_{1}^{5}\right) \\
& +\left(\varepsilon_{1} \mu_{2}+\varepsilon_{2} \mu_{1}\right)\left(\frac{3}{128} t_{1} t_{2}^{2}-\frac{11}{192} t_{1} t_{2}^{4}+\frac{35}{1152} t_{1} t_{2}^{6}-\frac{35}{1152} t_{1}^{6} t_{2}\right. \\
& +\frac{35}{3456} t_{1}^{6} t_{2}^{3}+\frac{11}{192} t_{1}^{4} t_{2}-\frac{11}{576} t_{1}^{4} t_{2}^{3}-\frac{35}{3456} t_{1}^{3} t_{2}^{6}-\frac{3}{128} t_{1}^{2} t_{2} \\
& +\frac{1}{128} t_{1}^{2} t_{2}^{3}+\frac{9}{128} t_{2}^{3}-\frac{293}{640} t_{2}^{5}+\frac{789}{1152} t_{2}^{7}-\frac{3115}{10368} t_{2}^{9}-\frac{1}{128} t_{1}^{3} t_{2}^{2} \\
& \left.+\frac{11}{576} t_{1}^{3} t_{2}^{4}+\frac{3115}{10368} t_{1}^{9}-\frac{9}{128} t_{1}^{3}-\frac{787}{1152} t_{1}^{7}+\frac{293}{640} t_{1}^{5}\right) \\
& +\varepsilon_{2} \mu_{2} \frac{t_{2}\left(1-t_{1}^{2}\right)}{t_{1}\left(1-t_{2}^{2}\right)}\left(-\frac{3}{128} t_{1} t_{2}^{2}+\frac{13}{64} t_{1} t_{2}^{4}-\frac{203}{1152} t_{1} t_{2}^{6}+\frac{205}{384} t_{1}^{6} t_{2}\right. \\
& -\frac{1435}{3456} t_{1}^{6} t_{2}^{3}-\frac{41}{64} t_{1}^{4} t_{2}+\frac{287}{576} t_{1}^{4} t_{2}^{3}+\frac{1015}{3456} t_{1}^{3} t_{2}^{6}+\frac{15}{128} t_{1}^{2} t_{2} \\
& +\frac{35}{384} t_{1}^{2} t_{2}^{3}+\frac{15}{128} t_{2}^{3}-\frac{1103}{640} t_{2}^{5}+\frac{469}{128} t_{2}^{7}-\frac{21385}{10368} t_{2}^{9}+\frac{5}{128} t_{1}^{3} t_{2}^{2} \\
& \left.\left.-\frac{65}{192} t_{1}^{3} t_{2}^{4}-\frac{22715}{10368} t_{1}^{9}+\frac{21}{128} t_{1}^{3}+\frac{4543}{1152} t_{1}^{7}-\frac{3671}{1920} t_{1}^{5}\right)\right] \\
& +\left(1-\eta^{2}\right) \eta^{2}\left(1-\alpha^{2}\right)^{2} \varepsilon_{2} \mu_{2} t_{1} t_{2}\left(\frac{5}{128} t_{1} t_{2}^{2}-\frac{41}{192} t_{1} t_{2}^{4}+\frac{205}{1152} t_{1} t_{2}^{6}\right. \\
& -\frac{205}{1152} t_{1}^{6} t_{2}+\frac{1025}{3456} t_{1}^{6} t_{2}^{3}+\frac{41}{192} t_{1}^{4} t_{2}-\frac{205}{576} t_{1}^{4} t_{2}^{3}-\frac{1025}{3456} t_{1}^{3} t_{2}^{6} \\
& -\frac{5}{128} t_{1}^{2} t_{2}+\frac{25}{384} t_{1}^{2} t_{2}^{3}-\frac{21}{128} t_{2}^{3}+\frac{3671}{1920} t_{2}^{5}-\frac{4543}{1152} t_{2}^{7}+\frac{22715}{10368} t_{2}^{9}
\end{aligned}
$$




$$
\begin{aligned}
& -\frac{25}{384} t_{1}^{3} t_{2}^{2}+\frac{205}{576} t_{1}^{3} t_{2}^{4}-\frac{22715}{10368} t_{1}^{9}+\frac{21}{128} t_{1}^{3}+\frac{4543}{1152} t_{1}^{7} \\
& \left.\left.-\frac{3671}{1920} t_{1}^{5}\right)\right\}
\end{aligned}
$$

and the denominator is

$$
\begin{aligned}
\mathcal{F}= & \frac{\left(1-t_{1}^{2}\right)\left(1-t_{2}^{2}\right)}{t_{1}^{2} t_{2}^{2}}\left[\varepsilon_{1} \mu_{1} \frac{t_{1}\left(1-t_{2}^{2}\right)}{t_{2}\left(1-t_{1}^{2}\right)}+\left(\varepsilon_{1} \mu_{2}+\varepsilon_{2} \mu_{1}\right)+\varepsilon_{2} \mu_{2} \frac{t_{2}\left(1-t_{1}^{2}\right)}{t_{1}\left(1-t_{2}^{2}\right)}\right] \\
& +\left(1-\eta^{2}\right) \eta^{2}\left(1-\alpha^{2}\right)^{2} \varepsilon_{2} \mu_{2} t_{1} t_{2}
\end{aligned}
$$

\section{References}

[1] M. Bordag, K. Kirsten, and D. Vassilevich. On the ground state energy for a penetrable sphere and for a dielectric ball. Phys. Rev., D59:085011, 1999.

[2] Kimball A. Milton and Y. Jack Ng. Observability of the bulk casimir effect: Can the dynamical casimir effect be relevant to sonoluminescence? Phys. Rev., E57:5504, 1998.

[3] G. Barton. Perturbative check on the casimir energies of nondispersive dielectric spheres. J. Phys., A32:525-535, 1999.

[4] V. V. Nesterenko, G. Lambiase, and G. Scarpetta. Casimir effect for a dilute dielectric ball at finite temperature. To appear in Phys. Rev. D, 2001. hep-th/0006121.

[5] K.A. Milton, A.V. Nesterenko, and V.V. Nesterenko. Mode-by-mode summation for the zero point electromagnetic energy of an infinite cylinder. Phys. Rev., D59:105009, 1999.

[6] M. Bordag and K. Kirsten. Vacuum energy in a spherically symmetric background field. Phys. Rev., D53:5753-5760, 1996.

[7] M. Bordag and K. Kirsten. The ground state energy of a spinor field in the background of a finite radius flux tube. Phys. Rev.,D60:105019, 1999. hep-th/9812060. 
[8] V. V. Nesterenko and I. G. Pirozhenko. Casimir energy of a compact cylinder under the condition epsilon $\mathrm{mu}=\mathrm{c}^{* *}(-2)$. Phys. Rev., D60:125007, 1999.

[9] M. Abramowitz and I.A. Stegun. Handbook of Mathematical Functions. Dover, New York, 1970.

[10] G. Barton. Perturbative casimir energies of dispersive spheres, cubes, and cylinders. Sussex Preprint, Nov. 2000.

[11] Valery N. Marachevsky. Casimir energy and dilute dielectric ball. 2000. hep-th/0010214.

[12] Valery N. Marachevsky. Casimir energy and realistic model of dilute dielectric ball. 2001. hep-th/0101062. 